\title{
Meteor showers associated with the near-Earth asteroid (2101) Adonis
}

\begin{abstract}
P. B. Babadzhanov ${ }^{\star}$
Institute of Astrophysics, Tajik Academy of Sciences, and Isaac Newton Institute of Chile, Tajikistan Branch; Bukhoro Str. 22, Dushanbe 734042, Tajikistan

Received 22 August 2002 / Accepted 19 September 2002

Abstract. The orbital evolution of the near-Earth asteroid (2101) Adonis under gravitational action of six planet (Mercury to Saturn) is investigated by the Halphen-Goryachev method. The theoretical geocentric coordinates and velocities of four possible meteor showers associated with this asteroid are determined. Using published data, the theoretically predicted showers are identified with the observed ones, namely, night-time $\sigma$-Capricornids and $\chi$-Sagittariids, and day-time $\chi$-Capricornids and Capricornids-Sagittariids. The existence of meteor showers associated with Adonis provides evidence supporting the conjecture that this asteroid may be of a cometary nature. The small 50-m near-Earth asteroid 1995 CS probably represents a large Adonis fragment and belongs to a part of the Adonis meteoroid stream, which produces the day-time $\chi$-Capricornids meteor shower.
\end{abstract}

Key words. comets: general - meteors, meteoroids - minor planets, asteroids

\section{Introduction}

According to the available data regarding Near-Earth Asteroids (NEAs), they may originate either from the main belt of asteroids or be extinct comets. The main mechanism removing asteroids from the main belt requires the help of gravitational resonances with the planets, especially Jupiter. Such NEAs may be expected to be stony or iron bodies.

Another part of the NEA population could be composed of extinct or dormant cometary nuclei. These NEAs are the end state of normal cometary nuclei after multiple perihelion passage, resulting in loss of all their available volatiles or being covered by a mantle that prevents sublimation of subsurface ice. Any NEA formed by this process would contain a substantial proportion of frozen volatiles and have a very weak structure. An extinct cometary nucleus hitting the Earth would produce events like the Tunguska fall. Thus, NEAs from both sources are hazardous for the Earth, though differently.

From groundbased observations of NEAs it is difficult to distinguish between the different origins. Hence it is very important to use any additional criteria which can help us to determine the nature of specific asteroids and identify them as "dead" cometary nuclei and to determine the fraction of extinct comets in the asteroid population. One of the possible criteria is the existence of meteoroid streams produced during the period of cometary activity.

Because a meteor shower can only be produced from the meteoroid stream that intersects the Earth's orbit, the search for dead comets through the use of associated meteoroid streams

\footnotetext{
* e-mail:pb@tajik.net
}

can only be meaningful when conducted within the NEA population. There are currently (August 5, 2002) 1947 NEAs (http://newton.dm.unipi.it/cgi-bin/neodys) and the number of newly discovered NEAs is increasing very rapidly. Up to now only a dozen asteroids have been shown to have associated meteor showers, most being the association of asteroid (3200) Phaethon and the Geminid meteor shower, and associations of Taurid complex asteroids and about 40 observable meteor showers (Babadzhanov \& Obrubov 1987; Babadzhanov 2001).

Adonis (2101) is one of the first NEAs discovered in 1936 by Delport and designated as 1936 CA. Some days before, it passed at a distance of $1.5 \mathrm{mln} \mathrm{km}$ from the Earth. For two months it was observed by the $2.5 \mathrm{~m}$ telescope at the MauntWilson observatory, and was lost afterwards. In 1977 Adonis was rediscovered and numbered as 2101. Presently, asteroid (2101) Adonis has the following orbital elements (2000.0):

Semimajor axis $a=1.874 \mathrm{AU}$.

Eccentricity $e=0.765$.

Perihelion distance $q=0.443 \mathrm{AU}$.

Inclination $i=1.35^{\circ}$.

Longitude of ascending node $\Omega=350.7^{\circ}$.

Argument of perihelion $\omega=42.3^{\circ}$.

Longitude of perihelion $(\pi=\Omega+\omega) \pi=33.0^{\circ}$.

The possible association of Adonis with the ScorpiidSagittariid meteor shower, based on the similarity of the orbital elements, was suggested by Hoffmeister (1947), but the calculation of the evolution of the orbit of this shower by Plavec $(1953,1954)$ did not confirm its association with Adonis. For the points of the minimum distances of the Adonis orbit from 
the Earth's orbit, Drummond (1982) calculated the theoretical geocentric radiants and velocities. According to the values of Southworth \& Hawkins' (1963) D-criterion, which is a measure of similarity of two orbits and is less than 0.2 for well established relations of meteor showers with comets, Drummond concluded that Adonis is associated with the $\chi$-Sagittariids and Scorpiids-Sagittariids meteor showers and that this asteroid seemed to be a defunct comet. Possible association of Adonis with 6 showers was assumed also by Sekanina (1976). Weissman et al. (1989) suggested that the NEAs Adonis and some others are very likely to be of cometary origin, and that their physical properties are consistent with this assumption. For example, anomalous radar echoes from (2101) Adonis (Ostro et al. 1991) had been regarded as suggestive that this asteroid may be a extinct comet (McFadden et al. 1993).

As shown earlier (Babadzhanov \& Obrubov 1992; Babadzhanov 1998, 2001), the number of meteor showers produced by a meteoroid stream corresponds to the Earth-crossing class of the parent-body orbit, since because of the initial dispersion of orbital elements of meteoroids ejected from parent body with different velocities, under the differencial perturbing action of major planets on meteoroids with different semimajor axes and eccentricities, the stream meteoroids occupy all evolutionary tracks of their parent body. During a year's orbiting around the Sun, the Earth collides with those stream meteoroids which have orbital nodes at the heliocentric distance $r \approx 1 \mathrm{AU}$, i.e. satisfying the expression:

$\omega \approx \pm \arccos \frac{a\left(1-e^{2}\right)-1}{e}$.

As seen from condition (1) for a given $a$ and $e$ the Earth's orbit may be intersected at four values of $\omega$. As a result, one meteoroid stream may produce two night-time showers at the pre-perihelion intersections and two day-time showers at the post-perihelion intersections with the Earth. For example, asteroid (3200) Phaethon is a quadruple crosser of the Earth's orbit and the meteoroids of the stream that separated from Phaethon, having various values of the argument of perihelion, can form four meteor showers: the pre-perihelion Geminids and Canis Minorids, post-perihelion Day-time Sextantids and $\delta$-Leonids (Babadzhanov \& Obrubov 1992).

In accordance with our concept of the evolution of meteoroid streams, for the investigation of possible genetic relationship between near-Earth objects and meteor showers we use a new method which includes the following operations (Babadzhanov \& Obrubov 1992; Babadzhanov 1998, 2001):

1) The calculation of the orbital evolutions of a nearEarth object (comet or asteroid) for a time interval covering one cycle of variation of the perihelion argument. The Halphen-Goryachev (Goryachev 1937) or Everhart's RADAU19 (Everhart 1974) methods and integrators are used.

2) The determination of the orbits crossing the Earth's orbit and of the Earth-crossing class of the parent body, i.e., the number of crossings during one cycle of variation of the perihelion argument. The number of crossings may be from one to eight.

3) The calculation of the theoretical geocentric radiants and velocities for the Earth-crossing orbits.
4) Search for theoretically predicted radiants in catalogs of observed meteor showers and of individual meteors.

The results of the search for meteor showers associated with asteroid (2101) Adonis are presented below.

\section{Search for meteor showers associated with Adonis}

We calculated the secular perturbations of the Adonis orbital elements using the Halphen-Goryachev method. Gravitational perturbations from six planet (Mercury-Saturn) were taken into account. The perturbations by other planets are very small, and are neglected. Results of calculations show that during the time interval embracing one cycle of variations of the argument of perihelion $(\sim 13000 \mathrm{yrs})$ Adonis intersects the Earth's orbit four times.

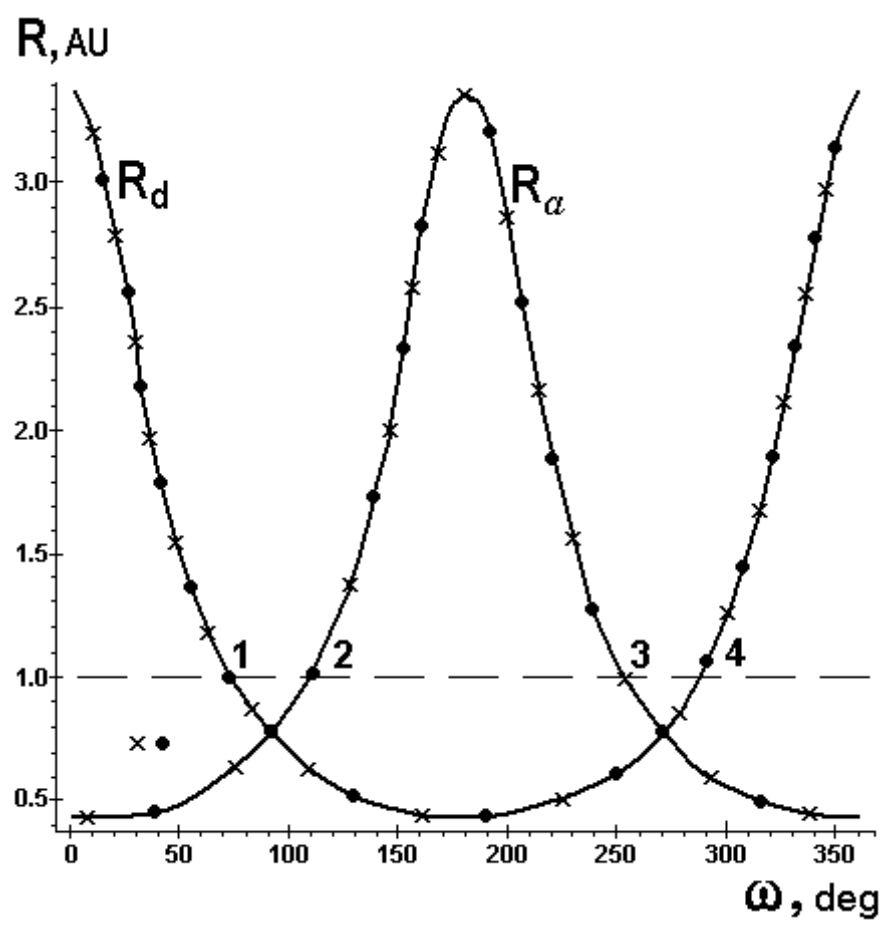

Fig. 1. Dependence of the radius-vectors of the ascending $\left(R_{\mathrm{a}}\right)$ and descending $\left(R_{\mathrm{d}}\right)$ nodes of the asteroids (2101) Adonis (dots) and 1995 CS (crosses) on the perihelion argument. Crossing 1 correspond to Capricornids-Sagittariids; crossing 2 to $\chi$-Sagittariids; crossing 3 to $\chi$-Capricornids; crossing 4 to $\sigma$-Capricornids.

Figure 1 shows the secular variations of the radii-vectors to the ascending $R_{\mathrm{a}}$ and descending $R_{\mathrm{d}}$ nodes of the Adonis' orbit as a function of the argument of perihelion $\omega$. As seen, Adonis crosses the Earth's orbit at the values of $\omega$ equal to $72^{\circ}, 105^{\circ}, 253^{\circ}$ and $287^{\circ}$, and therefore its possible meteoroid stream might produce four meteor showers. The theoretical orbital elements of the meteor showers associated with asteroid Adonis are presented in Table 1, and the theoretical geocentric coordinates of radiant (right ascention $\alpha$ and declination $\delta)$, the velocity $V_{g}\left(\mathrm{~km} \mathrm{~s}^{-1}\right)$, the dates activity and the solar longitudes $L_{\odot}$ corresponding to these dates are given in Table 2. 
Table 1. Theoretical $(\mathrm{T})$ and observed $(\mathrm{O})$ orbital elements of the meteor showers associated with Adonis.

\begin{tabular}{|c|c|c|c|c|c|c|c|c|c|c|}
\hline \multirow[t]{2}{*}{ Meteor showers } & & $\begin{array}{c}q \\
\mathrm{AU}\end{array}$ & $e$ & $i^{\circ}$ & $\begin{array}{c}\Omega^{\circ} \\
2000.0\end{array}$ & $\omega^{\circ}$ & $U$ & $\cos \theta$ & $D_{S-H}$ & Catalog \\
\hline & $T$ & 0.437 & 0.767 & 3.7 & 106 & 287 & 0.84 & -0.14 & & \\
\hline \multirow[t]{2}{*}{$\sigma-$ Capricornids } & $O$ & 0.431 & 0.758 & 2.1 & 107 & 290 & 0.84 & -0.16 & 0.01 & $S 1$ \\
\hline & $T$ & 0.442 & 0.764 & 0.2 & 288 & 105 & 0.84 & -0.14 & & \\
\hline \multirow[t]{2}{*}{$\chi-S$ agittariids } & $O$ & 0.430 & 0.783 & 3.9 & 280 & 108 & 0.86 & -0.14 & 0.02 & $S 2$ \\
\hline & $T$ & 0.437 & 0.767 & 3.6 & 140 & 253 & 0.84 & -0.14 & & \\
\hline$\chi$-Capricornids & $O$ & 0.355 & 0.789 & 6.8 & 145 & 243 & 0.91 & -0.23 & 0.02 & $S 1$ \\
\hline \multirow[t]{2}{*}{ 1995CS } & $\mathbf{O}$ & 0.439 & 0.769 & 2.6 & 136 & 252 & 0.84 & -0.14 & $\mathbf{0 . 0 3}$ & \\
\hline & $T$ & 0.437 & 0.767 & 0.2 & 321 & 72 & 0.84 & 0.14 & & \\
\hline $\begin{array}{l}\text { Capricornids- } \\
\text { Sagittariids }\end{array}$ & $O$ & 0.415 & 0.758 & 6.2 & 310 & 70 & 0.85 & -0.18 & 0.01 & $S 2$ \\
\hline
\end{tabular}

Table 2. Predicted (T) and observed (O) geocentric radiants and velocities of the meteor showers associated with (2101) Adonis. Type $\mathrm{N}$ is for night shower, type D for day shower. (Equinox 2000.0).

\begin{tabular}{lccccccccccc}
\hline \hline \multirow{2}{*}{$\begin{array}{l}\text { Meteor } \\
\text { showers }\end{array}$} & $L_{\odot}$ & Date & $\alpha^{\circ}$ & $\delta^{\circ}$ & $V_{g}$ & $L_{\odot}$ & Date & $\alpha^{\circ}$ & $\delta^{\circ}$ & $V_{\mathrm{g}}$ & Type \\
\hline$\sigma-$ Capricornids & 106 & Jul.8 & 296 & -18 & 25.2 & 107 & Jul. 9 & 299 & -18.7 & 24.6 & $N$ \\
--Sagittariids & 108 & Jul. 10 & 297 & -22 & 24.9 & 100 & Jul. 2 & 291 & -26.0 & 25.6 & $N$ \\
X-Capricornids & 320 & Feb. 10 & 316 & -21 & 24.9 & 325 & Feb. 15 & 315 & -23.3 & 26.8 & $D$ \\
1995CS & $\mathbf{3 1 6}$ & Feb. 5 & $\mathbf{3 1 1}$ & $-\mathbf{2 1}$ & $\mathbf{2 4 . 9}$ & & & & & & $D$ \\
Capricornids- & 321 & Feb. 11 & 314 & -17 & 24.9 & 310 & Jan. 31 & 300 & -14.2 & 25.1 & $D$ \\
Sagittariids & & & & & & & & & & & \\
\hline
\end{tabular}

Valsecchi et al. (1999) used the quantities of $U$ and $\cos \theta$ to identify as possibly originating from the same body, meteor showers with orbits having different $q, e, i, \Omega$ and $\omega$, due to secular perturbations on the same meteoroid stream. Here $U$ is the geocentric velocity of the meteoroids when crossing the Earth's orbit

$U=\sqrt{3-T}$,

where $T$ is the Tisserand parameter

$T=\frac{1}{a}+2 \sqrt{a\left(1-e^{2}\right)} \cos i$,

and $\theta$ is the elongation of the geocentric radiant from the apex, i.e. the angle between $U$ and the direction of motion of the Earth, which depends on $U$ and $a$

$\cos \theta=\frac{\left(1-U^{2}-\frac{1}{a}\right)}{2 U}$.

The values of $U$ and $\cos \theta$ are given in the seventh and eighth columns of Table 1 both for theoretically predicted and observed meteor showers associated with Adonis.

We undertook a computerized search for the predicted showers in the published catalogs of observed meteor showers: (C) Cook (1973), (K) Kashcheev et al. (1967), (L) Lebedinets et al. (1972), (S1, S2) Sekanina (1973, 1976), and (T) Terentyeva (1989) - their parenthesized notation is used in Table 1. This search took into account the closeness in the positions of the predicted and the observed radiant (requirement used was $\Delta \alpha=\Delta \delta \leq 10^{\circ}$ ), in velocity values $\left(\Delta V_{g} \leq 5 \mathrm{~km} \mathrm{~s}^{-1}\right)$ and period of activity $(\Delta t \leq 15$ days) for $D_{S-H}<0.2$, where $D_{S-H}$ is Southworth \& Hawkins' (1963) criterion, which serves as a measure of similarity of two orbits - in the case under consideration, as a measure of the similarity between the predicted and the observed orbits.

With the use of these data, all four theoretically predicted showers associated with the asteroid (2101) Adonis were identified with the observed showers: night-time $\sigma$-Capricornids and $\chi$-Sagittariids, daytime $\chi$-Capricornids and CapricornidsSagittariids. All of these showers are produced by the same meteoroid stream, which may consist of meteoroids of any possible value of the arguments of perihelia $\omega$.

Tables 1 and 2 list the observed (O) orbital elements, solar longitudes and corresponding dates of maximum activity, the geocentric coordinates of the radiants and velocities for all four showers. The values of $D_{S-H}, U$ and $\cos \theta$ given in the seventhnineth columns of Table 1 show good agreement between the theoretically predicted and the observed showers, i.e. all four possible Adonis' meteor showers are active to date. The existence of the meteor showers associated with Adonis provides evidence supporting the conjecture that this asteroid may be of a cometary nature. 


\section{Is asteroid $1995 \mathrm{CS}$ a fragment of Adonis?}

In February 1995, using the Spacewatch telescope at the Kitt Peak observatory, Jedicke (1995) discovered a very faint asteroid of an absolute magnitude $H=25$ when it passed near the Earth at a distance of about 2 million $\mathrm{km}$. The equivalent diameter $d$ of $1995 \mathrm{CS}$, calculated using the following expression (Bowell \& Lumme 1982)

$\log d=3.12-0.2 H-0.5 \log p$,

for a assumed albedo $p=0.08$, is equal to $50 \mathrm{~m}$, wheras that of Adonis is equal to $0.8 \mathrm{~km}$. It turned out that $1995 \mathrm{CS}$ has the following orbital elements (Marsden 1995):

$a=1.900 A U, e=0.769, q=0.439 A U$,

$i=2.60^{\circ}, \Omega=135.7^{\circ}, \omega=252.2^{\circ}, \pi=27.9^{\circ}$.

They are very similar to that of Adonis, with their directions of perihelion $(\pi=\Omega+\omega)$ differing by only about $5^{\circ}$, but their longitude of ascending nodes $(\Omega)$ and arguments of perihelion $(\omega)$ differ substantialy being in quite different phases of their precession cycles.

The similarity in the directions of perihelion, semi-major axes, eccentricity, and perihelion distances of these two objects imply that they separated from a common body at an earlier time or the smaller 1995 CS detached from the larger Adonis (Steel 1997). In order to investigate possible genetic relationship of the Adonis and 1995 CS, Steel (1997) performed a series of back integrations of the orbits of Adonis and $1995 \mathrm{CS}$ over the time span of $\sim 55000 \mathrm{yr}$ and came to the conclusion that these two objects might have had identical orbits at least $\sim 30000$ yr ago and therefore:

1) the small asteroid $1995 \mathrm{CS}$ may be a fragment of the larger body (2101) Adonis, which by then represented an extinct or dormant cometary nucleus, or

2) as for 1995 CS, Adonis is itself a fragment of a larger cometary body yet to be discovered, or that might be known but wandered into a sufficiently different orbit, or

3) it is possible that Adonis and 1995 CS do not share a common origin, but occupy similar orbits in the present epoch merely by chance.

Our back integration of the orbit of 1995 CS over $\sim 15000$ yr performed by the Halphen-Goryachev (Goryachev 1937) method shows that $1995 \mathrm{CS}$ is a quadruple-crosser of the Earth's orbit for the same values of the argument of perihelion ( $\omega$ ) as Adonis (Fig. 1). Moreover, it was found that the orbital elements of 1995 CS coincide nearly with the theoretical ones of day-time $\chi$-Capricornids (Table 1). Therefore it seems that 1995 CS belongs to the meteoroid stream associated with Adonis. At the time of its discovery, the radius-vector of the ascending node of $1995 \mathrm{CS}$ was equal to $1.0 \mathrm{AU}$, and so using 1995 CS' orbital elements, for the point of minimum distance from the Earth's orbit we calculated the theoretical geocentric radiant and velocity equal to:

$L_{\odot}=315.7^{\circ}, \alpha=310.8^{\circ}, \delta=-21.1^{\circ}, V_{g}=24.9 \mathrm{~km} \mathrm{~s}^{-1}$,

which correspond to the geocentric radiant and velocity of the day-time $\chi$-Capricornid meteor shower associated with Adonis (see Table 2). On these grounds we may conclude that 1995 CS probably is a fragment of Adonis, and belongs to the stream meteoroids producing $\chi$-Capricornid meteor shower.

The membership of 1995 CS to the day-time $\chi$-Capricornid meteor shower shows that meteoroid streams may be populated also by large bodies of several tens of meters in diameter. Therefore, small extinct comets might be searched for along the orbits of meteoroid streams during periods of meteor shower activity. This inference is confirmed by the detection of 17 objects of some meters to some tens of meters, which passed within a few million $\mathrm{km}$ of the Earth. They were observed by Barabanov et al. (2001) during the period of activity of the Capricornids, Perseids, Leonids, and Coma Berenicids meteor showers, near the radiant positions of these showers, using 60-cm and 1-m telescopes with CCD-cameras ST-6, at the Zvenigorod (Moscow district) and Simeiz (Crimea) observatories of the Institute of Astronomy, Russian Academy of Sciences.

\section{Conclusions}

Investigation of the orbital evolution of Adonis shows that Adonis is a quadruple Earth-crosser and, therefore, its hypothetical meteoroid stream might produce four meteor showers observable from Earth in February and July. A search of the theoretically predicted radiants in the catalogs of observed meteor showers show that all these showers, namely, night-time $\sigma$ Capricornids and $\chi$-Sagittariids and day-time $\chi$-Capricornids and Capricornids-Sagittariids, are active at present. The existence of the meteor showers associated with Adonis provides evidence supporting the conjecture that this asteroid may be of a cometary nature.

On February 2, 1995, during the period of activity of the $\chi$-Capricornids meteor shower, the small near-Earth asteroid 1995 CS passed near the Earth at a distance of 0.014 AU. The predicted geocentric radiant position and velocity of the nearest point of the $1995 \mathrm{CS}$ orbit nearly coincides with the predicted and observed geocentric radiant and velocity of the $\chi$ Capricornid meteor shower associated with Adonis. Probably, 1995 CS is a large fragment of Adonis, or both Adonis and $1995 \mathrm{CS}$, and the meteoroid stream producing four abovenamed showers, have a common origin, having detached from a large cometary body some tens of thousands of years ago.

Acknowledgements. The author would like to express his gratitude to the referee Dr. G.B. Valsecchi for useful comments which improved the paper. The annotations by the English copy editor of the A\&A are also appreciated.

\section{References}

Babadzhanov, P. B. 1998, Celest. Mech. \& Dynam. Astron., 69, 221 Babadzhanov, P. B. 2001, A\&A, 373, 329

Babadzhanov, P. B., \& Obrubov, Yu. V. 1987, Interplanetary Matter, ed. Z. Ceplecha, \& P. Pecina, Publ. Astr. Inst. Czecho-Sl. Acad. Sci., 2, 141

Babadzhanov, P. B., \& Obrubov, Yu. V. 1992, Celest. Mech. \& Dynam. Astron., 54, 111 
Barabanov, S. I., Zenkovich, A. D., Mikisha, A. M., \& Smirnov, M. A. 2001, Observations of large bodies in the meteor and fireball streams, in Near-Earth Astronomy of the XXI Century, Proc. of Conf., Zvenigorod 2001, May 21-25, Moscow, GEOS, 158

Bowell, E., \& Lumme, K. 1982, Colorimetry and Magnitudes of Asteroids, in Asteroids, ed. T. Gehrels (Tucson: Univ. Ariz. Press), 132

Cook, A. F. 1973, A working list of meteor streams, in Evolutionary and Physical Properties of Meteoroids, ed C. L. Hemenway, P. M. Millman, \& A. F. Cook, NASA SP-319, Washington, D. C., 183

Drummond, J. D. 1982, Icarus, 49, 143

Everhart, E. 1974, Celestial Mechanics, 10, 35

Hoffmeister, C. 1947, Meteorstrome (Verlag Johann Ambrosine Bart), Leipzig

Goryachev, N. N. 1937, Halphen's Method for Calculation of Planetary Secular Perturbations and its Application to Ceres (Krasnoe znamya, Tomsk)

Jedicke, R. 1995, MPEC 1995-C13

Kashcheev, B. L., Lebedinets, V. N., \& Lagutin, M. F. 1967, Meteoric Phenomena in the Earth's atmosphere (Nauka, Moscow)

Lebedinets, V. N., Korpusov, V. N., \& Sosnova, A. K. 1972, Trudy Inst. Eksper. Meteorol., No 1(34), 88
Marsden, B. 1995, MPC 24885

McFadden, L. A., Cochran, A. L., Barker, E. S., et al. 1993, J. Geophys. Res., 98. E2, 3031

Ostro, S. J., Campbell, D. B., Chandler, J. F., et al. 1991, AJ,102, 1490

Plavec, M. 1953, Bull. Astron. Inst. Czechosl., 4, 195

Plavec, M. 1954, Bull. Astron. Inst. Czechosl., 5, 38

Sekanina, Z. 1973, Icarus, 18, 253

Sekanina, Z. 1973, Icarus, 18, 253

Southworth, R. B., \& Hawkins, G. S. 1963, Smit. Contrib. Astrophys., 7, 261

Steel, D. I. 1997, Planet. Space Sci., 45, 327

Terentyeva, A. K. 1989, Fireball Streams. in Asteroids, Comets, Meteors III, ed. C.-I. Lagerkvist, H. Rickman, B. A. Lindblad, \& M. Lindgren (Uppsala Universitet, Reprocentralen HSC), Uppsala, 579

Valsecchi, G. B., Jopek, J. J., \& Froeschle, Cl. 1999, MNRAS, 304, 743

Weissman, P. R., A'Hearn, M. F., McFadden, L. A., \& Rickman, H. 1989, Evolution of Comets into Asteroids, in Asteroids II, ed. R. P. Binzel, T. Gehrels, \& M. S. Mathews (Tucson: Univ. of Arizona Press), 880 